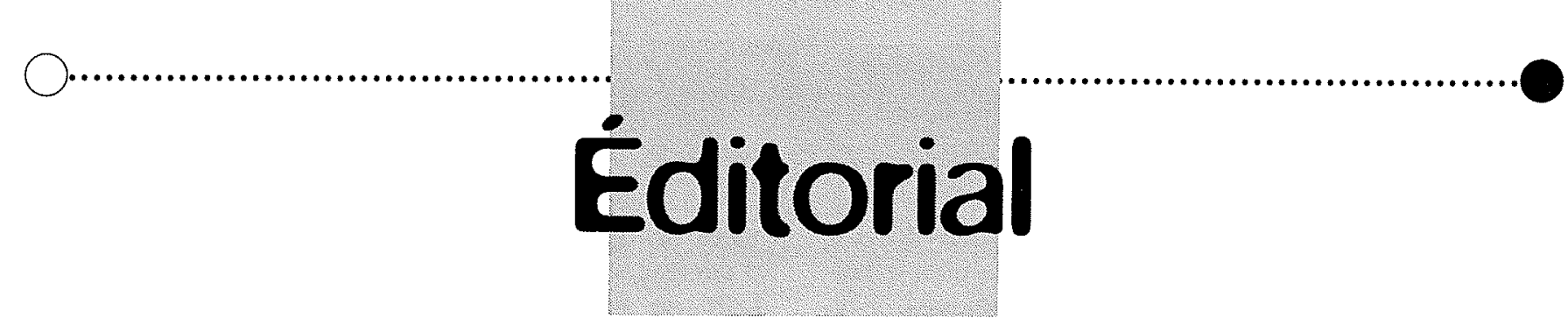

\title{
Halte au stress !
}

\author{
Jean-Louis LANG*
}

*Pédopsychiatre

Psychanayste,

Professeur associé à

I'Université Paris VII,

100 ,

rue de Rennes,

75006 Paris

France.

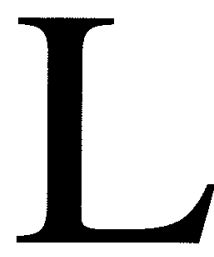

e stress est à la mode. Les médias en usent et en abusent, et pas seulement les médias. En l'identifiant à ce que 1'on a pu (à tort) décrire comme «stress psycho-émotionnel », elles en ont à la fois vulgarisé le mot et dévoyé le sens ${ }^{1}$.

Ainsi, par exemple, au hasard d'une écoute de radio ou de télévision, mais aussi bien d'une banale conversation: «J'ai du stress » (heureusement), ou même ... « le stress »; ... « il est en état de stress perpétuel $» . . . \ll$ il va de stress en stress »... «Untel a drôlement stressé dans l'attente du passage du vainqueur de la course $\gg .$. Tel autre est « en plein stress avant d'entrer en scène $\gg .$. Et quel potache, le jour de l'examen, ne se plaint pas - en s'esclaffant ou en tremblant, c'est selon les tempéraments - « d'avoir du stress, beaucoup de stress $\gg .$. ou pas du tout. Il n'est alors pas inutile de rappeler quelques vérités simples. Le stress, tel que le définit celui qui l'a caractérisé et décrit, Hans Selye, est une notion physiologique qui s'exprime à travers le système neuro-hormonal et qui constitue «une défense d'adaptation, d'ailleurs non spécifique, donnée par le corps à toute demande qui lui est faite [...], que l'agent provocateur soit plaisant ou désagréable $[. .$.$] , ce qui compte, c'est son intensité »$ (H. Selye); et ceci à côté d'autres systèmes défensifs, telles que les défenses immunologiques, réflexologiques, la douleur, etc. ${ }^{2}$

Ainsi, parler d'une «thérapeutique du stress », d'une «prévention du stress », est non seulement un contresens mais aussi une absurdité. C'est également un danger quand l'une ou l'autre sont sensées devoir s'appliquer à une maladie (une «maladie de la civilisation » a-t-on dit) dont, à la suite des médias et de l'opinion publique, les responsables de la Santé et de la Famille risquent de psychologiser, voire de psychiatriser, le contenu et les effets.

Et ce n'est pas en protestant que ce qui est ici visé ne serait pas le stress en lui-même mais ses effets que 1'on modifiera en quoi que ce soit ces abus de langage et surtout leurs conséquences pratiques.

Si j'insiste sur ces contresens, ces dangers, ces risques, c'est qu'en effet ils ne sont pas illusoires. J'ai eu récemment l'occasion d'en avoir des échos et d'en discuter au cours d'une Journée d'Études tenue, le 19 mars 2004, à 1'Hôpital de la Salpêtrière, dans le service du Pr Mazet. Cette Journée était organisée en l'honneur du Professeur Duché, Professeur émérite de psychiatrie infanto-juvénile, ancien titulaire de ce même service, la «Clinique Georges Heuyer », dont un département d'hospitalisation de jour pour enfants portera désormais le nom d'«Unité psychopédagogique D.J. Duché ».

Le thème de cette Journée était « La psychiatrie de l'enfant et de l'adolescent: hier, aujourd'hui, demain $\gg$. Au cours des divers exposés et discussions auxquels participèrent de nombreux orateurs (dont un Canadien, un Suisse et un Marocain), plusieurs d'entre eux évoquèrent les situations dites de stress, et notamment pour en dénoncer le mésusage et s'inquiéter des mesures préconisées par certains à leur sujet.

Sont ainsi mis en œuvre, dans des cas plus ou

\footnotetext{
1 Vulgarisation et déviation qui n'ont d'égales que celles de "psychanalyse », devenu synonyme d'analyse psychologique, voire de traduction d'un soit-disant inconscient, à tel point que J. Laplanche proposa de reprendre l'ancien terme de « psychoanalyse » pour en restreindre l'emploi aux seules théories et pratiques issues de la pensée freudienne. Mais comment pouvoir rendre au «stress » sa rigueur d'origine?

2 Je renvoie à ce propos à l'ouvrage de P. et H. Lôo [1].
} 
moins justifiés, des dispositifs « anti-stress », destinés à « lutter contre le stress » ou à le prévenir: «cellules » (?) psychologiques, psychiatriques ou sociales comportant psychologue, psychothérapeute, assistante sociale ou familiale, si possible un psychiatre; équipe appelée en urgence en cas d'accident sur la voie publique, à l'école ou en colonie, accident de car, noyade, disparition d'un enfant..., tous plus ou moins anxiogènes et spectaculaires, surtout s'ils concernent des enfants.

Certes, il est des catastrophes ou des drames où le recours à de telles cellules est amplement justifié et où, de fait, il s'agit de prévenir d'éventuelles conséquences tant chez les intéressés que dans leur entourage, ces traumatismes étant en effet susceptibles d'engendrer des défenses violentes, disproportionnées ou dépassant leur but (hyperanxiété, cauchemars, égarements, paniques de groupe...) et qui, chez certains sujets, enfants ou adolescents notamment, peuvent entraîner des séquelles durables d'ordre psychique (phobies, dépression, anorexie, arrêt des règles, troubles du comportement, tics...) relevant de la pédo-psychiatrie.

Mais ces cas ne sont pas si fréquents, surtout si on ne les met pas en vedette. Bien souvent, un éducateur, une institutrice, voire un psychologue scolaire ou un directeur d'établissement devront être aptes (à condition, bien sûr, d'en être informé et formé, ce qui devrait aller de soi) à consoler, calmer l'anxiété, apaiser et sécuriser le groupe, quitte à s'occuper plus particulièrement de certains. Les jours suivants, ils pourront donner aux enfants l'occasion de s'exprimer sur l'accident, de parler de ce qu'ils ont vécu, de leurs réactions, ou encore d'en dessiner les évocations; et parfois aussi de provoquer des rencontres avec les parents concernés. C'est alors d'ailleurs que pourrait se dévoiler les cas plus fragiles, suspects de troubles importants ou durables, qu'il conviendrait d'orienter vers un spécialiste ou, par exemple, un CMPP.

Insister sur le traumatisme, sur les effets spectaculaires de l'événement, les dramatiser, entraîne inévitablement une majoration de ses conséquences psychiques, contribue à le fixer et à en engendrer des séquelles. Je le répète, dans la majorité des cas, le personnel d'encadrement et les responsables naturels de l'établissement et de la famille devraient suffire à faire face à l'événement traumatisant et à en maîtriser les effets, sans recours à une «psychologisation » qui n'est pas sans risque. Nous manquons actuellement de pédopsychiatres et de psychologues cliniciens pour nos ser- vices de diagnostic et traitement d'enfants et d'adolescents présentant divers troubles mentaux ou carences psychiques exigeant des mesures curatives et préventives actives et souvent prolongées. L'appel à de soi-disant techniques psycho-sociales ou para-psychiatriques comme celles, ponctuelles, de la « lutte antistress », est alors fréquemment un luxe inutile qui ne fait que cacher, je ne dirai pas la misère, mais la lente détérioration de nos structures pédo-psychiatriques.

Alors, élargissons le débat: je dénoncerai deux courants de notre politique de santé, de santé mentale du moins, que j'estime pernicieux. Je viens d'en évoquer le premier : l'infiltration de la vie sociale par ce que j'ai nommé une psychologisation, une psychiatrisation, superficielles et illusoires, où 1'avis du psychologue ou du pédopsychiatre est sollicité à propos de tout et de rien ; ce qui n'engage ni celui qui le demande, ni celui qui y répond, mais bien plutôt le lecteur lui-même ou l'auditeur. C'est l'arbre qui cache une forêt mal portante. Le pédopsychiatre n'est ni un super-éducateur, ni un psycho-sociologue hautement qualifié, ni un confesseur agréé.

La seconde orientation à dénoncer est la politique qui vise à restreindre le nombre et les activités de certaines spécialités médicales qui pourraient être transférées à des généralistes «compétents», avec ou sans certificat d'Université. Ainsi en serait-il du gynécologue (généraliste de la femme), du pédiatre (généraliste de l'enfant), du psychiatre (généraliste du psychisme), du gériatre (généraliste du $3^{\mathrm{e}}$ ou du $4^{\text {e }}$ âg). Ainsi, la pédopsychiatrie, dissoute au sein d'une psychiatrie générale (adultes, enfants, adolescents, vieillards) tendrait-elle à disparaître, même si devaient subsister des services réservés à tel ou tel âge. Ce paraît être ainsi la position, partagée d'ailleurs par certains de nos collègues, d'une commission européenne sans doute séduite par le dogme de la maîtrise des dépenses de santé.

Pour notre discipline, cette évolution traduirait un fatal retour en arrière, avec une psychiatrie dite «riche» pour les cas relevant a priori d'une «thérapeutique active» (névroses, troubles réactionnels, « petits mentaux », maladies mentales à leur début...), quitte à confier les autres (les déments, les arriérés, les délirants chroniques, les autistes et les schizophrènes, les psychopathes aussi) à de nouveaux asiles, à de nouveaux aliénistes (psychiatrie «pauvre ») ; quitte également à orienter ceux qui présentent de «simples» troubles du comportement, de l'humeur ou de l'affectivité, s'il ne sont pas trop gênants, vers 
des psychologues praticiens, des éducateurs spécialisés, des psycho-pédagogues, et à abandonner les «cas sociaux graves», les « sauvageons », les délinquants, sinon parfois à la rue, à des services éducatifs ou répressifs, non thérapeutiques.

Bien sûr, nous sommes encore bien loin d'un nouveau «grand renfermement» et, pour revenir à notre sujet, il reste bien entendu que certaines «situations de stress » relèvent effectivement de «cellules » spécialisées et compétentes dont il est hors de question de faire le procès.

Ajoutons, cette fois d'un point de vue prophylactique, qu'il existe bien une prévention, non pas du stress, mais des conséquences, chez certains sujets, d'une sensibilité exagérée ou particulière à ce type de système physiologique défensif. Elle vise à en atténuer la rigueur et l'ampleur, à l'assouplir, à éviter ainsi des réactions brutales ou durables. Il en est ainsi des séances de relaxation, du yoga, de la balnéo- ou thalasso-thérapie, de certaines cures thermales...

Sans avoir à y recourir, un changement dans les orientations de la santé mentale contribuerait sûrement à restreindre les réactions «de stress $»$ de nos collègues pédopsychiatres.

\section{RÉFÉRENCE BIBLIOGRAPHIQUE}

1. Lôo P, Lôo H. Le stress permanent. Paris: Masson, 1986.

\section{Communiqués}

\section{Séminaire BABEL \\ Psychanalyse, littérature et arts \\ Année 2005}

Amphithéâtre de l'Institut Mutualiste Montsouris dans le hall d'accueil

Les lundis 13 juin, 27 juin

$20 \mathrm{~h} 30$ précise

Entrée $10 €$ - Étudiants $5 €$

\section{Renseignements}

Corinne Dugré-Le Bigre, Département de psychiatrie de l'Adolescent et du Jeune Adulte Institut Mutualiste Montsouris, 42, Boulevard Jourdan, 75014 Paris, France.

Tél. : 0156616919 -Fax:0156616918-Email : corinne.dugré-lebigre@imm.fr

\section{$2^{\mathrm{e}}$ colloque BABEL}

Samedi 18 juin 2005

\section{Lettre de l'extrême... Extrême de l'être}

Avec : R. Roussillon, J. Hochmann, M. Corcos, A. Rojas-Urrego, M. Gribinski...

\section{Renseignements}

Corinne Dugré-Le Bigre, Département de psychiatrie de l'Adolescent et du Jeune Adulte, Institut Mutualiste Montsouris, 42, boulevard Jourdan, 75014, Paris, France

Tél : 0156616919 .Fax:0156616918-E-mail : corinne.dugre-lebigre@imm.fr 